\title{
LOS CAMBIOS EN EL REGISTRO DE DEUDORES ALIMENTARIOS Y SU REGLAMENTO A PROPÓSITO DE LA EMISIÓN DEL DECRETO LEGISLATIVO N 1377.
}

CHANGES IN THE REGISTRY OF FOOD DEBTORS AND THEIR REGULATIONS FOR THE ISSUANCE OF LEGISLATIVE DECREE N¹377.

EMILIO José BALAREZo ReYes*

Magister en Derecho Civil y Comercial por la Universidad de San Martín de Porres. Abogado en Ejercicio. Catedrático de Derecho Civil de la Universidad de San Martín de Porres, Universidad Privada del Norte - Filial Lima y la Universidad Científica del Sur milobare@hotmail.com, 988010447 


\title{
LOS CAMBIOS EN EL REGISTRO DE DEUDORES ALIMENTARIOS Y SU REGLAMENTO A PROPÓSITO DE LA EMISIÓN DEL DECRETO LEGISLATIVO N 1377.
}

\author{
CHANGES IN THE REGISTRY OF FOOD DEBTORS AND THEIR REGULATIONS FOR THE \\ ISSUANCE OF LEGISLATIVE DECREE N`1377
}

Emilio José Balarezo Reyes

\begin{abstract}
RESUMEN:
Las recientes modificaciones que ha traído el Decreto Legislativo $\mathrm{N}^{\circ} 1377$ y su efecto en las instituciones como en las modalidades en las que se busca el cumplimiento de la institución de los alimentos lleva a analizar de nuevo la viabilidad de la norma y su respectivo reglamento como también la forma en que han venido siendo aplicadas en la realidad nacional. Solo con un replanteamiento adecuado creará el efecto deseado por sus creadores y su cumplimiento por parte de la sociedad y el rol que juega el Estado.
\end{abstract}

\section{PALABRAS CLAVE:}

Alimentos, pensión, deuda, morosidad, niños, adolescentes, conciliación, juez.

\begin{abstract}
:
The recent modifications that Legislative Decree No. 1377 has brought and its effect on institutions as well as on the modalities in which compliance with the food institution is sought leads us to analyze again the viability of the regulation and its respective regulations as also the way in which they have been applied in the national reality. Only with an adequate rethinking will it create the desired effect by its creators and its compliance by society and the role played by the State.
\end{abstract}

\section{KEY WORDS:}

Food, pension, debt, delinquency, children, adolescents, Conciliation, Judge.

\section{Introducción.}

Dentro de las múltiples repercusiones que ha tenido el Decreto Legislativo 1377, Decreto que fortalece la Protección Integral de Niñas, Niños y Adolescentes hay un eje temático ligado sobre todo a las responsabilidades ineludibles e inherentes como básicas y trascendentales que tienen los padres que es el cumplimiento de los alimentos, en las fechas y momentos oportunos de acuerdo a lo que ha dictaminado el Juez en caso sea resultado de un proceso que buscaba ese fin como también del cumplimiento del acuerdo llevado a cabo mediante una Conciliación Extrajudicial, en el cual los acuerdos tomados en la correspondiente Acta de Conciliación resulta incumplida.

Ambos mecanismos de carácter netamente procedimental tienen entre ellos un mismo mensaje buscan tutelar uno de los derechos más inherentes que tiene el ser humano que no solo se circunscribe al ámbito de proveer los insumos para la preparación de la comida para el sostenimiento del menor sino que compete muchos más aspectos que efectivamente están inmersos en el concepto de "Alimentos" ya que esta institución lo que busca es lograr que el proceso de formación del ser humano sea de carácter integral, en torno al esparcimiento, vestimenta, etc. Así es importante señalar lo que expresa Varsi cuando expresan lo siguiente: "Todos requerimos de alimentos para poder vivir estos incluyen educación, vivienda, salud, vestido, recreación y todo lo necesario para 
el pleno desarrollo e integración de la persona en sociedad. Es un derecho por demás fundamental correspondiéndole al Estado exigir su cumplimiento" (Varsi, 2011, p.104)

Creemos que la dación del Decreto Legislativo 1377, decreto que fortalece la Protección Integral de Niñas, Niños y Adolescentes, de una revisión preliminar se aprecia que lo que busca es otorgar un orden, mayor organicidad como sistemática a la norma que regula el Registro de Deudores Alimentarios (REDAM), un papel relevante es la inclusión de las disposiciones para la realización del pago y las modalidades que se presentan para el desarrollo de las mismas con el objetivo de incentivar la inserción como la continuidad laboral de la persona responsable de llevar a cabo el pago de los alimentos.

\section{Análisis Sistemático del Registro de Deudores Alimentarios (REDAM)}

Desde su dación en enero del año 2007 mediante la Ley $N^{\circ} 28970$ y su Reglamento por medio del Decreto Supremo N ${ }^{\circ}$ 002-007-JUS, el Registro de Deudores Alimentarios ha sufrido en todo este tiempo de vigencia múltiples comentarios tanto positivos como negativos los mismos que han buscado resaltar las consecuencias que ha tenido la presente norma en el ordenamiento jurídico, respecto a su conocimiento, a su uso como a su aplicación. Una realidad en la cual muchas veces este registro no refleja la realidad y por ende no responde a los objetivos planteados para los que había sido creado. Tomando en cuenta que desde su dación no ha disminuido en los más mínimo el porcentaje de procesos de alimentos como también la cantidad de personas que han sido sentenciadas con el pago de los mismos; los cuales han hecho caso omiso a las consecuencias que podrían derivarse de esta situación, creando un clima de desconfianza respecto a la utilidad de esta norma conjuntamente con su reglamento. Como lo señala López: "la dación de la ley de creación del Registro de Deudores Alimentarios Morosos obedece al papel tuitivo del Estado frente al drama de las personas que necesitan de una pensión alimentaria, más aún cuando no solo el Estado sino la sociedad es consciente de los problemas que afrontan los acreedores alimentarios para ver satisfechas sus expectativas alimentarias" (López, 2007, p.19)

En sí el espíritu de la norma es netamente procedimental, ya que describe el trámite netamente administrativo que se deriva del incumplimiento del deber alimentario como primer ítem destacable esta la forma en la cual la persona que lleva a cabo la omisión aliméntaria ingresa a este registro, estableciéndose claramente que por el incumplimiento de tres (03) cuotas sean estas continuas o no producto de un proceso previo en el cual se dio la sentencia respectiva, otra modalidad por la cual se ingresa a ese registro es aquella que se deriva del acuerdo conciliatorio al que llegan las partes como producto de una Conciliación sea esta judicial o extrajudicial, ambas tanto la Sentencia como el Acta de Conciliación deben tener carácter de cosa juzgada; una tercera forma mediante la cual se puede ingresar es por la no cancelación de devengados producto de las cuotas que no han sido canceladas en su debido momento cuando estas tienen la categoría de exigibles. Por su parte el doctor Campana plantea lo siguiente respecto a este registro: "conscientes de la impostergable necesidad de no cejar en el intento de protección de nuestra minoridad creemos que la creación del Registro de Deudores Alimentarios resulta una tarea urgente, obligatoria y forzosa" (Campana, 2006, p.13)

El rol del Poder Judicial, se centra en la función que tiene el Órgano de Gobierno de esta institución, la cual a través de la Gerencia sostiene los dos roles puntuales que este tiene en la responsabilidad del Registro de Deudores Alimentarios - REDAM, el primero en elaborar un consolidado de todos aquellos deudores que han caído en morosidad en el cumplimiento de sus sentencias, actas de consolidación a la que llegaron como resultado del acuerdo en la transacción efectuada entre las partes con el fin de cumplir dentro de sus posibilidades con el pago de los alimentos, segundo, la expedición de los denominados "Certificados de Registro" con la finalidad de esclarecer al existir alguna duda si se encuentra o no una persona dentro de la misma, donde se deberá consignar sus generales de ley, fotografía, el monto adeudado y el órgano jurisdiccional que ordenó el pago. Aparte de lo expresado 
procedimentalmente en la norma el ámbito técnico en lo correspondiente a la implementación de este registro también recae en la Geréncia del Órgano de Gobierno del Poder Judicial.

La importancia del Libro del Registro de Deudores Morosos Alimentarios radica en la información que contiene, la misma que tiene que estar constantemente actualizada debiendo ser el reflejo situacional actual de los deudores. El procedimiento previo a la inscripción se sostiene en un procedimiento previo con plazos mínimos en los cuales se solicitan el cumplimiento de lo adeudado al moroso y se le informa del proceder qué se llevará a cabo en caso de incumplir con esta situación; la única forma de salir y no encontrarse dentro de este registro es cumpliendo y a su vez solicitar administrativamente el levantamiento de la inscripción y por ende la condición de deudor registrado, la actualización es mensual y el acceso al mismo es gratuito.

Una situación particular es la remisión de la información a las Centrales de Riesgo sean estas privadas como también a la Superintendencia de Banca, Seguros y Administradora de Fondo de Pensiones, también señala la existencia de un cruce de información por parte del Ministerio de Trabajo y Promoción del Empleo cuando remite en lista la información de los Contratos de Trabajo que han sido firmados para poder ubicar a los Deudores Morosos registrados, pero no es la única institución del Estado que sigue este rol de colaboración para un mayor seguimiento y control de los deudores la SUNARP - Superintendencia Nacional de los Registros Públicos, también remite a la Gerencia una relación de las transferencias realizadas por las personas naturales en lo que respecta a los bienes muebles o inmuebles que han sido transferidos con la finalidad de ubicar y verificar la existencia de patrimonio por parte de los deudores. El ingreso a las bases de datos respectivos debe de realizarse vía electrónica

Es de destacar la responsabilidad del Funcionario Público que omita a sabiendas de la existencia de un deudor moroso en lo que respecta a la información en torno a la ubicación de los Deudores Morosos los mismos que pueden caer en una situación de falta administrativa con su respectiva sanción como es el caso de la destitución.

A una visión panorámica de la norma se le podría atribuir su carácter de transversalidad en lo que respecta a los diferentes aspectos que este abarca como es resaltar la intervención del Estado en el cumplimiento de los objetivos como son el seguimiento y el de sanción administrativa en razón de la intervención, no solo del Poder Judicial sino de diferentes organismos vinculados desde una óptica patrimonial al tema como son el Ministerio de Trabajo y la Superintendencia Nacional de los Registros Públicos.

Del entendimiento de las ideas como de los aportes que nos da el Reglamento de la presente Ley el mismo que se dio mediante el D.S 002-2007-JUS el 22 de marzo del 2007, como todo reglamento cumple con la finalidad de ampliar asi como esclarecer los puntos que han sido tocados en la Ley, en su estructura encontramos en el inicio del texto las definiciones de las diferentes figuras que son acotadas en la norma, sobre lo cual vamos a tocar a nuestro concepto los tres principales y que a continuación pasamos a desarrollar:

El primero es la definición de Deudor Alimentario Moroso, esta categoría se le da a la persona ya sancionada como consecuencia de un proceso en donde ha podido ejercer su derecho de defensa, la cual tiene la obligación de prestar alimentos, otro aspecto concurrente es que se adeude tres cuotas ya sean sucesivas o alternadas, un segundo concepto es el de Registro de Deudores Alimentarios Morosos, al cual se le otorga la categoría de Libro Electrónico, el cual es actualizado con la información que proviene del Poder Judicial en lo que respecta a sus datos y su condición, aquí debemos destacar el papel que juega la información proporcionada por el Poder Judicial ya que si no se cuenta con la misma, la actualización como el control y seguimiento del estado situacional de cada una de las personas que se encuentran inmersas dentro de esta, no logrará el objetivo de conminarlo al pago 
de lo adeudado, debido que es público, provoca un rechazo de parte de la sociedad, y al obtener la información tanto laboral, económica como patrimonial de la persona que se encuentra dentro del registro lo ubica en una situación bastante especial frente al mercado laboral e inclusive con la finalidad de evitar problemas posteriores, los empleadores pueden determinar su "inelegibilidad"; debemos destacar entonces el rol de la información como también su utilidad.

Un último concepto que desde nuestra percepción es relevante es la de Certificado de Registro Positivo o Negativo, creemos desde nuestra óptica que este documento juega un rol importante pues es el reflejo material de la información que se encuentra en el Registro de Deudores Alimentarios Morosos, este certificado podemos entenderlo como sumamente importante ya que el mismo determina la condición en la que se encuentra la persona frente al registro y constituye un elemento muchas veces dirimente ya que los centro laborales o determinadas instituciones exigen o podrían exigir la presencia indubitable de los mismos con el objetivo de verificar la idoneidad de la persona en su rol como padre o madre en el cumplimiento de una de sus principales responsabilidades que tiene como tal como es el de proveer a su hijo o hijos sean la condición que estos tengan de todo lo concerniente a lo que abarca la institución de los "Alimentos" el cual no debe ser únicamente circunscrito a la comida sino que abarca otros aspectos como el esparcimiento, la educación, vestimenta, etc.

Como lo hemos señalado la utilidad de este Registro recae en la información que este contiene en la actualización de la misma y en el seguimiento respectivo del estado y condición de todas las personas que se encuentran dentro del mismo, recayendo esta responsabilidad en el Consejo Ejecutivo del Poder Judicial, el servicio, diligencia y cuidado de la información como de la actualización de la misma e allí su importancia y utilidad, ya que de no contar con las condiciones adecuadas para su uso y continuidad idóneos, el mismo caerá en una situación de poca credibilidad como de poca confianza por parte de la población al querer recurrir a la información que obra en este Registro.

La Declaración Judicial de Deudor Moroso, tendrá necesariamente la concurrencia de tres figuras, la solicitud de la parte beneficiaria de los alimentos, teniendo esta condición como resultado de un proceso terminado y sacramentado jurídicamente, es decir existiendo decisión fundamentada por el órgano jurisdiccional respectivo, esta condición debe ser acreditada con la falta de pago de tres cuotas sucesivas o alternadas, acá el mensaje es la existencia del incumplimiento, la solicitud a la que se hace referencia al inicio es un instrumento netamente formal y lo que busca es la uniformidad y el orden al momento de llevar a cabo el registro del estado situacional del deudor, por último se hace acotación que esta situación puede ser apelada es decir, la persona que ha sido incluida en el registro de Deudores Morosos ejerce sus facultades y derechos para variar su situación; en este caso lo más relevante es demostrar que ha cumplido con los pagos en las fechas y montos respectivos, mientras que se evalúa su apelación no impide a que se proceda su inscripción en este registro, situación que puede variar posteriormente.

Respecto al procedimiento, el criterio que ha seguido la norma es desarrollar esta parte consignando las generales de ley de todo ciudadano en torno a sus datos como es el tema del nombre, domicilio, el documento nacional de identidad, el número de expediente del proceso de alimentos, el nombre de la persona beneficiada con los alimentos que no ha recibido, los mismos o en su defecto los ha recibido de manera no continua y en cantidades inapropiadas para crear las condiciones mínimas de desarrollo para el beneficiario; concluye esta parte con la referencia a la fotografía para una identificación visual del deudor, para lo cual se recurre a otra institución del estado como es la RENIEC - Registro Nacional de Identidad y Estado Civil, el cual también proveerá de la dirección donde vive el deudor, estos datos son categorizados como confiables debido a que son proporcionados por la institución ad hoc que ve estos temas.

Un aspecto a nuestro criterio relevante es el tema de la Cancelación del Registro, ya que a través de la misma se pone fin a la inclusión dentro de la misma de las personas que se encuentran en 
calidad de deudores, lo que hay que destacar es que a la misma única y exclusivamente se llega mediante un mandato judicial es decir mediante una respectiva resolución emanada del órgano jurisdiccional el cual busque ese fin, al haber demostrado el cumplimiento como también el pago de todo lo adeudado, se hace énfasis también en señalar que jamás se debe recurrir a la vía administrativa con el objetivo de lograr este fin, ahora la exigibilidad proviene del interesado ya que si continúa dentro del registro su situación en el mercado laboral como también frente a la sociedad no será la más cómoda ni tampoco la más idónea para su desarrollo normal debe de buscar el levantamiento respectivo de esta situación.

Los artículos tanto 7 y 8 del Reglamento se van a sustentar en las comunicaciones que de manera periódica con la finalidad de establecer un puente constante de información se llevará a cabo entre el Órgano responsable del Registro como es el Consejo del Órgano del Poder Judicial y la Superintendencia de Banca, Seguros y AFP - SBS, como también con las Centrales Privadas de Información de Riesgo con el objetivo de que se inscriba en ellos mediante el registro respectivo a las personas que tienen la condición de deudores alimentarios; así el Sistema Financiero estará informado de la cantidad como de la identidad de las personas que se encuentran en calidad de deudores para proceder a la realización de los actos que correspondan como también a tomar las medidas del caso en las transacciones que realicen con la finalidad de evadir la responsabilidad que les corresponda.

Continúa el Reglamento señalando la obligatoriedad que se dará por parte de dos instituciones como son el Ministerio de Trabajo y Promoción del Empleo - MINTRA y la Superintendencia Nacional de los Registros Públicos -SUNARP, el primero para llevar a cabo la actualización y seguimiento de la información en lo que respecta a su situación laboral y se proceda a los descuentos en la planilla con la finalidad de que la decisión judicial que ha sido tomada en el caso de los alimentos se cumpla; esta situación puede traer complicaciones a los deudores, ya que en muchos casos las empresas no contratan a personas que se encuentran en esta condición con la finalidad de evitar problemas que puedan surgir posteriormente. En el caso de la SUNARP el tema es más patrimonial y se sostiene en el flujo patrimonial que tiene la persona que se encuentra en calidad de deudor moroso lo cual luego de la evaluación respectiva se podría proceder a establecer las medidas procesales como sustantivas que se crean convenientes con el objetivo de alcanzar asegurar el pago de la deuda alimentaria.

Un punto interesante es como ha manejado este tema el Sector Público recayendo la responsabilidad de esta situación en las Oficinas de Personal o de la sección que haga sus veces con el objetivo de llevar a cabo los filtros y cruce de información en torno a la condición de la personas que ingresan a laborar a este sector, los cuales al momento de ingresar firman una declaración judicial donde dejan constancia de su estado situacional en lo que respecta a las deudas alimentarias que tuvieran o no, esto en la búsqueda de la idoneidad del personal que labora dentro del Sector Público y en aras de la transparencia y honestidad de los datos consignados.

En el ámbito de las responsabilidades o roles que juega el Órgano Jurisdiccional debemos de destacar el de ser expeditivo como también de llevar a cabo las acciones correspondientes en aras de cumplir los objetivos de la ley y el reglamento como son la tramitación de medidas cautelares de existir así como de las resoluciones que sean necesarias.

Culmina el reglamento haciendo acotación a la difusión, creemos que este es un tema clave debido a que muchos de los comentarios negativos que se han dado respecto a esta norma se sostienen en el desconocimiento de la existencia de este registro como del contenido de la información que este tiene, su uso a veces es desconocido por la sociedad la cual no conoce las consecuencias de encontrarse o no registrado como también de hacer valer sus derechos y la concretización del rol de protección por parte del estado. Lo que se debe buscar es el acercamiento de la sociedad a la presente Ley como a su reglamento, por ende esta responsabilidad es compartida específicamente en tres instituciones el Poder Judicial, lo que era antes el MIMDES hoy Ministerio de la Mujer y 
Poblaciones Vulnerables y por último el Ministerio de Justicia y Derechos Humanos, los cuales deben llevar a cabo campañas coordinadas de difusión que busquen que la sociedad conozca y use la presente norma, situación que vemos todavía no se ha plasmado totalmente.

3.-El efecto de los cambios producidos por el DL. 1377 - Decreto que fortalece la Protección Integral de Niñas, Niños y Adolescentes en la Ley y el Reglamento del Registro de Deudores Alimentarios.

Como consecuencia de las modificaciones que se han dado con la dación del DL 1377 se han producido cambios en los artículos 3, 4 y 7 además se ha agregado el Artículo 10 a la Ley, los cuales a continuación pasaremos a analizar:

\section{Artículo 3.- Contenido del Registro de Deudores Alimentarios Morosos -REDAM-}

El Registro de Deudores Alimentarios Morosos debe contener la siguiente información:

a) Nombres y apellidos completos del Deudor Alimentario Moroso.

b) Domicilio real del Deudor Alimentario Moroso.

c) Número del Documento Nacional de Identidad u otro que haga sus veces, del Deudor Alimentario Moroso.

d) Fotografía del Deudor Alimentario Moroso.

e) Cantidad de cuotas en mora parcial o total, monto de la obligación pendiente e intereses hasta la fecha de la comunicación.

f) Indicación del órgano jurisdiccional que ordena el registro.

De la apreciación del contenido del actual Artículo 3, se puede apreciar que se han producido dos aspectos básicos y evidentes que son materia de reflexión, el primero es la salida del texto del concepto de lo que es el Registro de Deudores Alimentarios Morosos - REDAM, situación con la que estamos de acuerdo debido a que encontramos el desarrollo de esa definición en el Reglamento de la presente ley, a su vez denotamos que en la nueva estructura del presente artículo después del cambio va de manera directa a los elementos estructurales del Registro de Deudores Alimentarios Morosos no desarrollándose definición alguna en el actual contenido del artículo a diferencia de cómo estaba esbozado en la anterior redacción del texto, deja de lado entonces las conceptualizaciones convirtiéndose en un artículo que de forma directa abarca el contenido de los datos que deben estar consignados en el Registro de Deudores Alimentarios Morosos - REDAM.

Como lo hemos señalado, el cambio sustancial que se ha podido apreciar del contraste que se lleva a cabo a diferencia del texto original es que no se hace acotación el inicio de la tramitación para la inscripción de una determinada persona en el Registro de Deudores Alimentarios - REDAM este punto era netamente procedimental y el mismo no estaba adecuadamente ubicado, por lo que la redacción actual es más directa al solo señalar los requisitos para la inscripción sin hacer mención alguna a trámite alguno.

\section{Artículo 4.- Procedimiento}

4.1 El órgano jurisdiccional que conoce o conoció la causa, de oficio o a petición de parte y previo a ordenar la inscripción, notifica al obligado alimentario, para que informe en el 
término de tres (3) días el cumplimiento de la deuda. El juez ordena la inscripción en el mismo plazo si el deudor no demuestra el cumplimiento de la deuda o no absuelve el requerimiento.

4.2 El deudor puede oponerse a la inscripción o solicitar la cancelación de la inscripción solo si acredita haber cumplido con el pago de la deuda alimentaria. Dicha oposición o solicitud puede formularse en cualquier momento y tiene como efecto la cancelación de la inscripción.

4.3 En los casos de omisión de asistencia familiar, el juez penal informa al Registro de Deudores Alimentarios Morosos los datos de la persona procesada, para su inscripción correspondiente.

4.4 Cuando se solicite la oposición o cancelación de la inscripción, el Juez resuelve el levantamiento de la inscripción en un plazo máximo de tres (3) días.

4.5 Para los fines de la inscripción o cancelación de la inscripción en el Registro, el juez deberá oficiar al órgano de Gobierno del Poder Judicial en un plazo no mayor de tres (3) días luego de resolver la cuestión. El Órgano de Gobierno del Poder Judicial realiza la inscripción o cancelación en un plazo máximo de tres (3) días.

Encontramos en el nuevo texto del Artículo 4 de la ley una mejor organización y distribución en lo que respecta a su contenido desarrollándolo ordenadamente a través de incisos, que en total son cinco; anteriormente estaba consignado el presente tema de una forma general, cabe destacar que en el inciso primero se ha transcrito de forma literal a como estaba consignado en el texto original, es a partir del inciso segundo en donde se lleva a cabo las modificaciones, destacándose dentro del mismo que la única forma de que no proceda la inscripción es demostrando que la persona a la que se le imputa el pago de los alimentos lo ha llevado a cabo no expresando plazo alguno para poder llevar a cabo esta figura es decir, no existe limitación en lo que respecta al tiempo de su presentación; podemos concluir diciendo que sí reconoce el derecho de oposición con la finalidad de contrastar o no la existencia de la deuda por la cual se desea ingresar en el Registro de Deudores Alimentarios.

Un tema novedoso que ha sido incorporado es el de la intervención del Juez en lo penal, el cual podrá remitir los datos de la persona que se encuentra en un proceso por el Delito de Omisión de Asistencia Familiar para que se proceda a su inscripción en el Registro, con la finalidad de llevar a cabo la tramitación de forma diligente y rápida la norma expresa que al haber presentado la persona a la cual se le atribuya la omisión de los alimentos las pruebas necesarias que ameriten lo contrario, el Juez tiene un plazo de tres (03) días para resolver es decir, en un tiempo breve y rápido con la intención de esclarecer el estado situacional de la persona supuestamente incumplida, para liberarla de todo tipo de sanción y sacarlo de la lista de Deudores Morosos, este trámite concluye con la comunicación mediante Oficio por parte del Juez al Órgano de Gobierno del Poder Judicial, en este caso el Consejo Ejecutivo del Poder Judicial que tienen bajo su responsabilidad la actualización de los datos del Registro Nacional de Deudores Morosos - REDAM, para que procese al levantamiento de la inscripción o a su continuidad dentro del mismo.

Artículo 7.- Deber de colaboración entre las instituciones del Estado.

7.1 El Ministerio de Trabajo y Promoción del Empleo, a pedido del Órgano de Gobierno del Poder Judicial y en el plazo de cinco (5) días hábiles, remite la información de la planilla electrónica que resulte pertinente de los contratos laborales vigentes, de las personas inscritas en el REDAM, con la finalidad de comunicar a los juzgados correspondientes, en el término de la distancia, para que procedan conforme a sus atribuciones. 
7.2 La Superintendencia Nacional de los Registros Públicos, a pedido del Órgano de Gobierno del Poder Judicial, remite las listas de transferencias de bienes muebles o inmuebles registrables realizados por las personas inscritas en el REDAM, en un plazo de cinco (5) días hábiles.

7.3 El Banco de la Nación, en los casos que las pensiones alimenticias sean abonadas en cuentas administradas por dicha entidad, a pedido del Órgano de Gobierno del Poder Judicial, remite el reporte de abonos realizados a las personas inscritas en el REDAM, en un plazo de cinco (5) días hábiles.

7.4 El Órgano de Gobierno del Poder Judicial, al término de cada mes, debe remitir a las entidades señaladas en los numerales precedentes, el listado de personas inscritas en el REDAM, para el cumplimiento de la remisión de información.

Respecto al deber de colaboración de las instituciones para llevar a cabo de forma sigilosa el seguimiento como también el acompañamiento de las actividades laborales como económicas, en un primer análisis podemos observar que se desarrolla una mejor distribución de los temas que están contenidos dentro del presente artículo el cual está distribuido en (04) cuatro incisos, el primero de ellos tiene literalmente la misma redacción que antes de la modificación, en el cual se denota la función que cumple el Ministerio de Trabajo y Promoción del Empleo - MINTRA, el cual en el plazo de cinco días remite una vez que sea requerida la información laboral de las personas que se encuentren dentro del Registro de Deudores Morosos -REDAM, procederá a remitirla a los Juzgados correspondientes para que actúen conforme a sus funciones es decir, emitan comunicación mediante oficio y soliciten al empleador la retención del porcentaje respectivo por el cual han sido sancionados para que se lleve a cabo el pago de los alimentos pendientes la premura del pedido como de las acciones se justifica por que la institución de los alimentos es ineludible a su vez que necesaria para toda persona que lo solicita.

En situación similar la Superintendencia Nacional de los Registros Públicos - SUNARP remite la información al cabo de cinco (05) días de solicitada por el Órgano del Poder Judicial que tiene bajo su responsabilidad la gestión del registro, expresamos que esta información servirá para que la parte solicitante al conocer de la existencia de bienes que pertenecen en propiedad al deudor alimentario se proceda a llevar a cabo las acciones del caso para realizar sobre ellos las figuras jurídicas pertinentes como son los gravámenes o medidas cautelares que se estime convenientes para asegurar el pago de los alimentos que se solicitan.

Una de las novedades es la participación del Banco de la Nación como órgano encargado de remitir periódicamente al Consejo Ejecutivo del Poder Judicial la información sobre los pagos a través de abonos que ha venido realizando la persona que encuentra en calidad de registrado, con la finalidad de llevar a cabo un seguimiento en la forma en la que se está cumpliendo lo dictaminado por el juzgado luego del proceso respectivo.

Por último, se aprecia el rol del Órgano que tiene bajo su responsabilidad el Registro de Deudores Morosos de remitir el listado de las personas que se encuentren en situación de deudores alimentarios con la finalidad de viabilizar y hacer más dinámica como rápida la búsqueda de esta forma del cruce de la información remitida se podrá obtener resultados en el menor tiempo posible con el objetivo de desarrollar las acciones del caso en búsqueda del cumplimiento de todo lo pendiente por parte del deudor.

Como tema final hay que señalar que se incorporó el artículo 10 a la Ley $\mathrm{N}^{\circ} 28970$, Ley que crea el Registro de Deudores Alimentarios Morosos, en los siguientes términos:

Artículo 10.- Pago de la deuda alimentaria e incumplimiento. 
10.1 La persona inscrita en el Registro de Deudores Alimentarios Morosos puede postular y acceder al servicio civil en el Estado, o ser designado funcionario o directivo de confianza, o contratar con el Estado, siempre que cancele el registro o autorice el descuento por planilla o por otro medio de pago del monto de la pensión mensual fijada en el proceso de alimentos, previo a la suscripción del contrato o la expedición de la resolución de designación correspondiente.

10.2 La oficina de recursos humanos o la oficina de logística, según corresponda, o la que cumpla dichas funciones, comunica al REDAM la autorización de descuento, dentro del plazo de tres (3) días hábiles, bajo responsabilidad. La misma oficina se encarga de tramitar el depósito judicial respectivo a través de la oficina de tesorería o la que haga sus veces en la entidad, salvo disposición distinta del juzgado competente sobre la forma de pago.

10.3 En el sector privado, la autorización del descuento por planilla o por otro medio de pago del monto de la pensión mensual fijada en el proceso de alimentos se establece de común acuerdo entre el empleador y el trabajador o la persona postulante, debiendo la oficina de recursos humanos o la que cumpla dichas funciones, informar al REDAM de la autorización de descuento, dentro del plazo de tres (3) días hábiles.

El presente artículo constituye una novedad respecto al texto anterior, y nos hace referencia en el primer inciso a las facilidades que da ahora el Estado con la finalidad de no perjudicar a la persona que desea trabajar dentro de las instituciones públicas ya sea en cargo de confianza o no, el presente inciso da dos opciones a través de las cuales se pueda honrar el monto de la deuda contraída como resultado de los alimentos no pagados. El primer camino es autorizar los descuentos respectivos en lo que respecta al sueldo o pago que recibirá como contraprestación por el trabajo desarrollado y la otra es cancelar con los medios que cuente la deuda contraída con el alimentista, todo lo indicado debe desarrollarse antes de que se formalice la incorporación como trabajador dentro del Estado, a través de la firma del respectivo contrato o de la Resolución respectiva, de ser el caso, en que es nombrado funcionario de confianza dentro del Estado.

En cada institución del Estado, la Oficina de Recursos Humanos o la que haga sus veces dentro de la misma se encargará de comunicar al Registro de Deudores Alimentistas Morosos, la aceptación por parte del Deudor Moroso Alimentario de efectuar los descuentos respectivos en lo referido a su sueldo para honrar las deudas contraídas por los alimentos impagos, el depósito judicial con el que se concretiza el pago se llevará a cabo por concepto de alimentos y estará a cargo del área de tesorería de la institución, lo cual deberá ser informado en un plazo de tres días, es decir en un plazo breve en búsqueda de obtener resultados inmediatos los cuales pueden desencadenar la liberación del ex deudor, para su normal desarrollo en el ámbito laboral como económico.

En lo concerniente al Sector Privado, de manera precisa lo que se va a valorar es el acuerdo al que lleguen el empleador y el trabajador para proceder al pago de la deuda alimentaria en cuotas acordadas entre ellas, lo cual deberá ser comunicado de manera rápida y en el breve plazo de tres (03) días teniendo bajo su responsabilidad la transmisión de la comunicación a la oficina de recursos humanos o quien cumpla sus veces al Registro de Deudores Alimentarios Morosos de la autorización del deudor de la aceptación del respectivo descuento.

De lo analizado, se puede observar que los caminos que se están otorgando para el cumplimiento de la deuda cada vez son más accesibles y lo que buscan no es perjudicar al deudor en su desarrollo personal como profesional, pero sí que lleve a cabo las acciones pertinentes en búsqueda del respectivo pago con la finalidad de solventar la falta de recursos que tiene con el alimentista que al encontrarse en un estado de carencia y necesidad debe cumplir para poder asegurar su desarrollo y el alcance de sus objetivos dentro de la sociedad. Como lo acota Silva: "Sin restar importancia al 
registro en sí, es necesario recalcar que otro aporte de la normatividad que lo crea es el circuito de comunicación periódica e intercambio y cruce de información entre el Poder Judicial e instituciones del Estado para que los deudores sean mejor identificados y puedan ser exigidas sus obligaciones" (Silva, 2008, p.3)

\section{Conclusiones}

1.-La presente norma ha buscado desde su dación, viabilizar las formas en las cuales se lleva a cabo un seguimiento como también el cumplimiento de todas aquellas personas que se encuentran en calidad de deudores alimentarios, ya que esta situación común en nuestra sociedad muchas veces ha traído ante el porcentaje elevado de deudores, que este tipo de figuras no sean tomadas como vías de solución sino simplemente sea tomado como una figura de referencia.

2.-La necesidad de la participación de otros sectores del estado como la Superintendencia Nacional de los Registros Públicos -SUNARP y el Ministerio de Trabajo y Promoción del Empleo MINTRA nos refleja le necesaria interconexión entre las instituciones que conforman el Estado con la finalidad de un solo objetivo amparar y proteger a todas aquellas personas que se encuentran en calidad de alimentistas y que necesitan de forma urgente y rápida una solución a la situación por la que atraviesan.

3.-El seguimiento patrimonial y económico que se desarrolla a través de la Superintendencia de Banca y Seguros como a las Centrales de Riesgo en las cuales permiten hacer un seguimiento especializado de esta clase de deudores ya que muchas veces estos llevan a cabo actividades financieras y económicas de forma directa o a través de terceros con la finalidad de evadir sus responsabilidades como son el pago de los alimentos de forma oportuna y puntual.

4.-Los cambios que se han producido dentro de la norma han buscado otorgarle en primer lugar, más orden en el desarrollo de las instituciones que se dan tanto dentro de la ley como en el reglamento en la cual, la participación de los empleadores sean estos particulares como en el estado lo que buscan es crear los caminos a través de los cuales se pueda cumplir con los alimentos y de esta manera honrar los compromisos adquiridos con el alimentista.

5.-La continuidad como la salida del Registro de Deudores Alimentarios Morosos, dependerá del cumplimiento de la deuda, la cual se dará mediante la autorización del órgano jurisdiccional que llevó a cabo el proceso de alimentos que derivó en la inscripción en el respectivo registro, lo novedoso es que no se busca impedir el desarrollo ni laboral ni personal del deudor por lo que se le ha otorgado las facilidades del caso con la finalidad que cumpla con el pago de los alimentos.

\section{Referencias Bibliográficas}

- Campana, M (2006) Creación del Registro Nacional de Deudores Alimentarios en Jurídica $N^{\circ} 98$ Suplemento del Diario Oficial el Peruano.

- López, G (2007) ¿Resulta constitucional la implementación del Registro de Deudores Alimentarios Morosos? en Actualidad Jurídica N¹59 - febrero 2007.

- Silva, C (2008) En marcha el registro de deudores morosos en Jurídica Nº 184 Suplemento del Diario Oficial el Peruano.

- Varsi, E y Claudia Canales (2011) Deudores al extremo, el Registro de Deudores Alimentarios Morosos - REDAM en Libro de Ponencias del VI Congreso Nacional de Derecho Civil, Lima, Grandez Gráficos. 